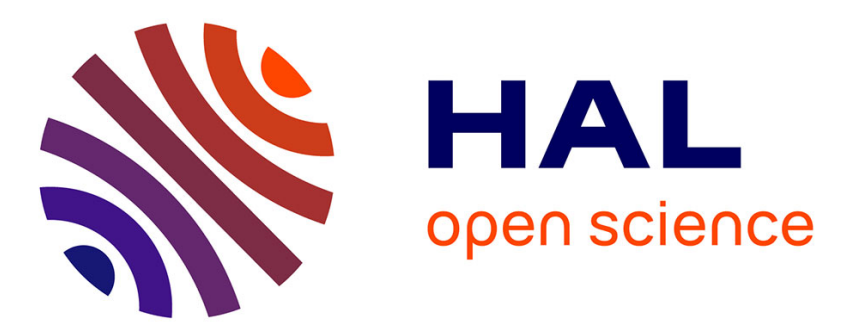

\title{
Applying the virtual fields method to determine the through-thickness moduli of thick composites with a nonlinear shear response
}

Michel Grédiac, François Auslender, Fabrice Pierron

\section{- To cite this version:}

Michel Grédiac, François Auslender, Fabrice Pierron. Applying the virtual fields method to determine the through-thickness moduli of thick composites with a nonlinear shear response. Composites Part A: Applied Science and Manufacturing, 2001, 32, pp.1713-1725. 10.1016/S1359-835X(01)00029-X . hal-00111340

\author{
HAL Id: hal-00111340 \\ https://hal.science/hal-00111340
}

Submitted on 29 Nov 2018

HAL is a multi-disciplinary open access archive for the deposit and dissemination of scientific research documents, whether they are published or not. The documents may come from teaching and research institutions in France or abroad, or from public or private research centers.
L'archive ouverte pluridisciplinaire HAL, est destinée au dépôt et à la diffusion de documents scientifiques de niveau recherche, publiés ou non, émanant des établissements d'enseignement et de recherche français ou étrangers, des laboratoires publics ou privés. 


\title{
Applying the virtual fields method to determine the through-thickness moduli of thick composites with a nonlinear shear response
}

\author{
Michel Grédiac ${ }^{\mathrm{a}, *}$, François Auslender ${ }^{\mathrm{b}, 1}$, Fabrice Pierron ${ }^{\mathrm{c}, 2}$ \\ ${ }^{a}$ Laboratoire d'Etudes et de Recherches en Mécanique des Structures, Université Blaise Pascal Clermont II, 24 avenue des Landais, BP 206, \\ 63174 Aubiere Cedex, France \\ ${ }^{\mathrm{b}}$ Laboratoire de Mécanique des Solides, UMR 7649, Ecole Polytechnique, 91128 Palaiseau Cedex, France \\ ${ }^{\mathrm{L}}$ Laboratoire de Mécanique et Procédés de Fabrication, Ecole Nationale Supérieure d'Arts et Métiers, rue Saint-Dominique, BP 508, \\ 51006 Châlons-en-Champagne, France
}

This paper presents a method allowing the simultaneous identification of parameters governing an orthotropic law with a nonlinear shear response. Such laws appear for instance through the thickness of thick laminated composites. The tested specimen is subjected to boundary conditions similar to those of a Iosipescu setup. The strain field in the central area is processed with the so-called virtual fields method, which is an application of the principle of virtual work with particular virtual fields. The method is simulated with data obtained from finite element calculations.

Keywords: B. Mechanical properties; Elastic properties

\section{Introduction}

Designing composite structures and predicting their mechanical behaviour requires the use of representative models together with accurate procedures to determine the parameters involved. However, this challenge is not easy to face in the case of composite materials because of the number of independent parameters to be determined. This problem becomes critical when through-thickness properties are concerned because usual tests are not easily adaptable to the through-thickness properties. These difficulties are underlined in Refs. [1-4] for instance. Nevertheless, the through-thickness properties are required when thick composite structures are designed. Such thick structures tend to appear nowadays in more and more industrial applications [5]. This is the consequence of moving from secondary to primary load bearing components, requiring more strength and therefore leading to the use of thicker composites.

\footnotetext{
* Corresponding author. Tel: +33-4-73-40-75-29; fax: +33-4-73-40-7494.

E-mail addresses: grediac@lermes.univ-bpclermont.fr (M. Grédiac), auslender@1ms.polytechnique.fr (F. Auslender), fabrice.pierron@chalons.ensam.fr (F. Pierron).

${ }^{1}$ Tel: +33-1-69-33-33-19; fax: +33-1-69-33-30-26

${ }^{2}$ Tel: +33-3-26-69-26-89; fax: +33-3-26-21-32-04
}

A solution for better measurement of mechanical properties of composites is to use one of the alternative methods based on mixed experimental/numerical procedures that have been developed during the last decade [6-16]. The main advantage of such approaches relies on the fact that several parameters can be determined from one single coupon. Moreover, the experimental set-up is allowed to develop heterogeneous strain/stress fields in the tested specimen since closed-form solutions are not necessary. This extra freedom can lead to simpler experimental setups. Furthermore, these heterogeneous strain/stress fields may involve simultaneously all the parameters governing the mechanical behaviour. Therefore, it should be possible to determine them provided that a suitable and efficient identification strategy is used.

Several approaches have been proposed to identify the elastic parameters of composite plates by back analysis of experimental data without any closed-form solution. Most of them are based on optimisation procedures with finite element calculations performed until the experimental data match the corresponding calculated ones. However this approach exhibits some drawbacks. For instance, the set of parameters input to initiate the optimisation procedure must often be close to the actual ones to guarantee convergence. Some difficulties are also expected to simulate the boundary conditions correctly, particularly if they are 
not point supports or forces. Finally, it must be pointed out that using a finite element programme is suitable for solving the direct problem of finding the displacement/strain/stress fields when the material properties are known. However, such a tool is not necessarily the best when the inverse problem of finding the material parameters from strain/ displacement fields is addressed.

The basic idea is here to consider the whole strain field onto the surface of the tested specimen as input data, i.e. to process many tens of thousands of experimental points. In practice, such fields can be measured with some suitable optical method. These methods are becoming more and more popular [17], but these experimental aspects are not considered in the present work. It has been shown in some recent studies that heterogeneous strain fields can be advantageously processed with a method called the virtual fields method which is simply based on the global equilibrium of the tested specimen written with the principle of virtual work. Feeding some particular virtual fields into this equilibrium equation leads in the case of elastic properties to a set of linear equations that are inverted to obtain the unknown parameters. This method, introduced first in Ref. [18], has been successfully applied to bending (either in statics [19,20], or in dynamics [21,22]), to in-plane $[23,24]$ and more recently to through-thickness composite characterization $[25,26]$.

In the present paper, the objective is to investigate the capability of the virtual fields method to identify parameters governing the nonlinear shear behaviour of a composite. The identification of parameters governing nonlinear material behaviour from mechanical configurations giving rise to heterogeneous stress/strain fields has seldom been addressed in the literature, even though some recent papers propose suitable strategies for the simultaneous identification of non elastic mechanical parameters of isotropic materials [27] for instance. Here, the aim is to consider an unnotched specimen tested in a Iosipescu fixture. Then, heterogeneous stress/strain fields take place in the central part of this specimen though shear/bending is predominant. In fact, the Iosipescu test used for shear characterization can provide extra modulus values by taking advantage of the presence of normal stresses in the gauge section. It is clear that no closed-form solution is available in this case, but one can expect to extract the whole set of unknown linear and nonlinear parameters with the virtual field methods, since a similar study limited to the linear response has been recently successfully carried out [26].

The virtual fields method presently used is recalled in the first part of the paper. The identification of the whole set of through-thickness moduli as well as the parameter governing the nonlinear shear response is then presented. The relevance of the approach is finally illustrated by some numerical simulations.

\section{Theoretical background}

\subsection{Global equilibrium of the tested specimen with the principle of virtual work}

Let us consider the tested specimen in a Iosipescu fixture, as shown in Fig. 1. Its equilibrium can be written with the principle of virtual work

$\int_{V} \sigma_{i j} \epsilon_{i j}^{*} \mathrm{~d} V=\int_{\partial V} T_{i} u_{i}^{*} \mathrm{~d} S$,

where $V$ is the volume of the specimen, $\mathrm{d} V$ the elementary volume element, $\mathrm{d} S$ the elementary outer surface element, $\sigma$ the stress field, $\epsilon^{*}$ the virtual strain field, $T$ the external load density, $u^{*}$ the virtual displacement field associated to $\epsilon^{*}$ and $\partial V$ is the boundary of the specimen.

The above equation is valid for any admissible virtual field $u^{*}$. The basic idea is to write the above equation with a set of different and independent virtual fields. Each new virtual field provides a new equation in which the parameters governing the material behaviour can be considered as unknown. This equation, if the constitutive equations of the material are assumed to have polynomial expressions, is linear with respect to the parameters that define the constitutive equations. Inverting the linear system provides the parameters. The main advantage of this approach is the fact that the unknowns are directly determined, without any updating of a finite element model, provided that the whole strain field onto a large part of the tested specimen is available. In practice, such a field can be obtained with a suitable optical technique, either directly [28] or by differentiation of the measured

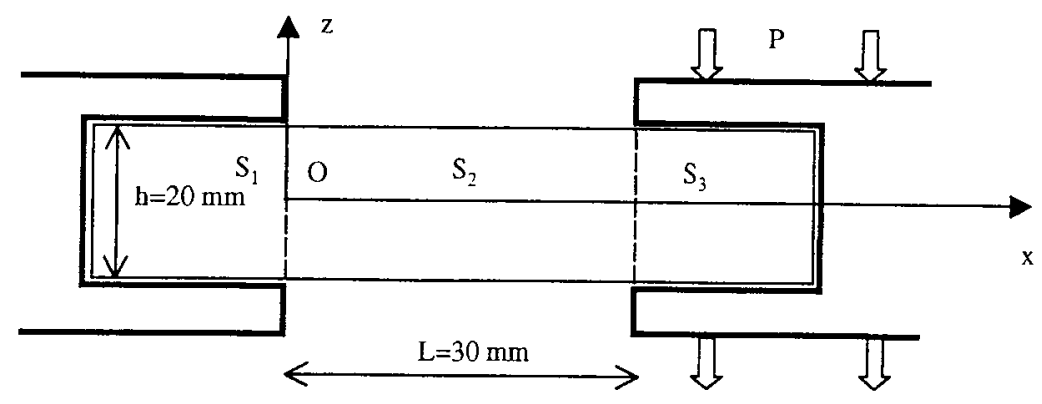

Fig. 1. Dimensions of the specimen. 


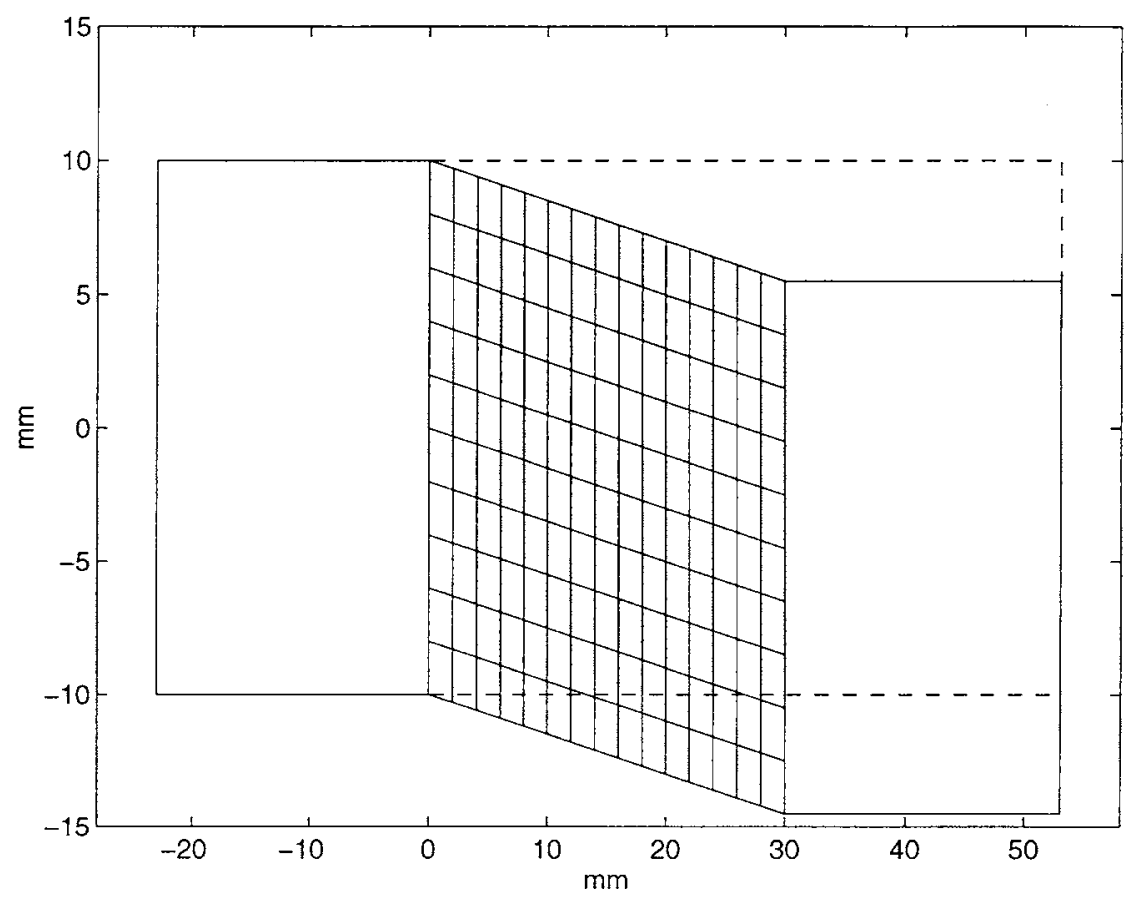

Fig. 2. Virtual field 1: constant virtual shear.

displacement field [29]. The first requirement for obtaining the parameters is the fact that heterogeneous strain/ stress fields take place in the tested specimen in such a way that all the unknowns influence the mechanical response of the specimen. Secondly, a set of as many independent virtual fields as mechanical parameters to be identified must be found in order to built up a system of equations where the mechanical parameters are unknown. Another way for obtaining new independent equations is to process actual strain fields provided by other mechanical configurations. These two possibilities are discussed below.



Fig. 3. Virtual field 2: virtual Euler-Bernoulli-like bending. 


\subsection{Linear response}

Let us considered as a first example the linear throughthickness orthotropic behaviour of a thick composite

$$
\left(\begin{array}{c}
\sigma_{x} \\
\sigma_{z} \\
\sigma_{r}
\end{array}\right)=\left(\begin{array}{ccc}
Q_{x x} & Q_{x z} & 0 \\
Q_{x z} & Q_{z z} & 0 \\
0 & 0 & Q_{r r}
\end{array}\right)\left(\begin{array}{c}
\epsilon_{x} \\
\epsilon_{z} \\
\epsilon_{r}
\end{array}\right),
$$

where $r$ holds for $x z$. Hence, $\epsilon_{x}=\epsilon_{x x}, \epsilon_{y}=\epsilon_{y y}$ and $\epsilon_{r}=$ $\gamma_{x z}=2 \epsilon_{x z}$. In this case, Eq. (1) becomes

$$
\begin{aligned}
& Q_{x x} \int_{S_{2}} \epsilon_{x} \epsilon_{x}^{*} \mathrm{~d} S+Q_{z z} \int_{S_{2}} \epsilon_{z} \epsilon_{z}^{*} \mathrm{~d} S \\
& \quad+Q_{x z} \int_{S_{2}}\left(\epsilon_{x} \epsilon_{z}^{*}+\epsilon_{z} \epsilon_{x}^{*}\right) \mathrm{d} S+Q_{r r} \int_{S_{2}} \epsilon_{r} \epsilon_{r}^{*} \mathrm{~d} S=\frac{P u_{z}^{*}(L)}{e},
\end{aligned}
$$

where $u_{z}^{*}(L)$ is the constant virtual displacement of surface $S_{3}$ (see Fig. 1) and $P$ is the global positive applied load on the right-hand side grip. It has been shown in Ref. [26] that a linear system of four equations can be built-up with the four virtual fields reported in Table 1 and plotted in Figs. 2-5. The first displacement field stands for a virtual constant shear of the central part of the specimen, which lies between the two grips (see Fig. 2). This part of the specimen is
Table 1

Four virtual fields used for the identification of the stiffnesses (after Ref [26])

\begin{tabular}{lllll}
\hline $\begin{array}{l}\text { Virtual } \\
\text { field \# }\end{array}$ & $u_{x}^{*}$ & $u_{z}^{*}$ & $u_{z}^{*}(L)$ & Area \\
\hline 1 & 0 & $-x$ & & \\
2 & $6 x z(L-x)$ & $x^{2}(2 x-3 L)$ & $-L$ & $S_{2}$ \\
3 & 0 & $x z(x-L)$ & 0 & $S_{2}$ \\
4 & 0 & $x z\left(x-\frac{L}{3}\right)$ & 0 & $A_{1}$ \\
& 0 & $z(x-L)\left(x-\frac{2 L}{3}\right)$ & 0 & $A_{2}$ \\
& 0 & 0 & 0 & $S_{2}-A_{1}-A_{2}$ \\
\hline
\end{tabular}

called henceforth 'active area'. The second virtual field leads to a virtual Euler-Bernoulli-like bending of the active area. The third one results in a virtual global compression of the active area. The fourth one finally results in a virtual local compression of two sub-areas: $A_{1}$ and $A_{2}$. Inverting the system of four equations obtained with these virtual fields leads to a direct identification of the mechanical parameters [26]. The $Q_{x z}$ stiffness is rather sensitive to noise in the data, but this feature is also underlined in other global identification procedures described in the literature.

\subsection{Nonlinear shear response}

As explained above, the objective is here to consider that the shear response is no more linear, but nonlinear. The idea is to consider the following stress/strain

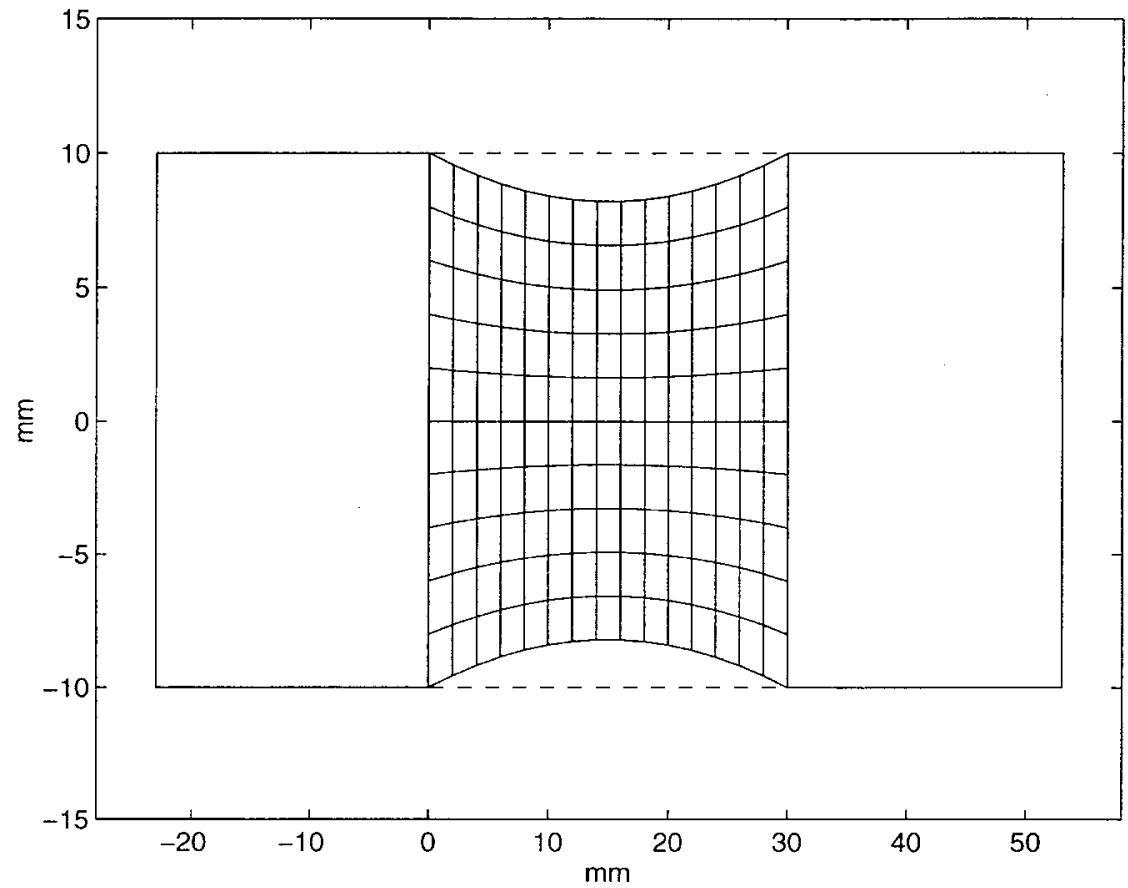

Fig. 4. Virtual field 3: virtual global compression. 


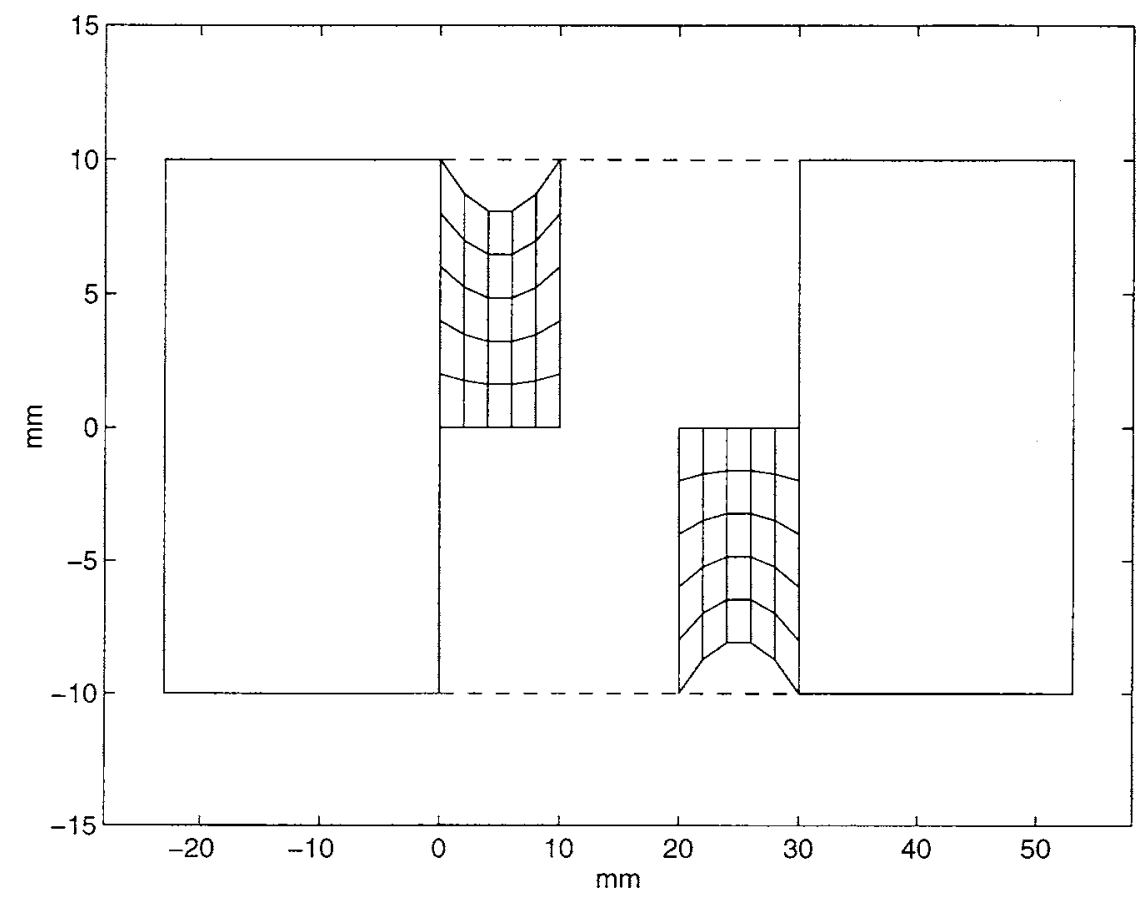

Fig. 5. Virtual field 4: virtual local compression.

relationship

$\sigma_{r}=Q_{r r} \epsilon_{r}-K \epsilon_{r}^{3}$

where $K$ is a positive real number. Such a polynomial law models correctly the shear behaviour of composites [30-32]. Feeding the above law in Eq. (1) leads to

$$
\begin{aligned}
& Q_{x x} \int_{S_{2}} \epsilon_{x} \epsilon_{x}^{*} \mathrm{~d} S+Q_{z z} \int_{S_{2}} \epsilon_{z} \epsilon_{z}^{*} \mathrm{~d} S \\
& \quad+Q_{x z} \int_{S_{2}}\left(\epsilon_{x} \epsilon_{z}^{*}+\epsilon_{z} \epsilon_{x}^{*}\right) \mathrm{d} S+Q_{r r} \int_{S_{2}} \epsilon_{r} \epsilon_{r}^{*} \mathrm{~d} S \\
& \quad-K \int_{S_{2}} \epsilon_{r}^{3} \epsilon_{r}^{*} \mathrm{~d} S=-\frac{P u_{z}^{*}(L)}{e} .
\end{aligned}
$$

It is worth noting that the polynomial law in Eq. (4) describing a nonlinear behaviour leads to a linear equation where the five material parameters are unknown. Other types of law would lead to nonlinear equations and would require more sophisticated numerical tools for the resolution. Since a polynomial law is presently used, the objective is to build up a linear system of five independent equations. Two ways can be explored for constructing this system. At first, one can try to find five different virtual fields associated with only one actual testing configuration. In this case, the system writes

$$
\mathbf{A X}=\mathbf{B},
$$

where $\mathbf{A}$ is a matrix and $\mathbf{B}$ a vector such that

$$
\left\{\begin{array}{l}
\mathbf{A}_{i 1}=\int_{S_{2}} \epsilon_{x} \epsilon_{x_{i}}^{*} \mathrm{~d} S \\
\mathbf{A}_{i 2}=\int_{S_{2}} \epsilon_{z} \epsilon_{z_{i}}^{*} \mathrm{~d} S \\
\mathbf{A}_{i 3}=\int_{S_{2}}\left(\epsilon_{x} \epsilon_{z_{i}}^{*}+\epsilon_{z} \epsilon_{x_{i}}^{*}\right) \mathrm{d} S \\
\mathbf{A}_{i 4}=\int_{S_{2}} \epsilon_{r} \epsilon_{r_{i}}^{*} \mathrm{~d} S \\
\mathbf{A}_{i 5}=\int_{S_{2}} \epsilon_{r}^{3} \epsilon_{r_{i}}^{*} \mathrm{~d} S \\
\mathbf{B}_{i}=-\frac{P u_{z_{i}}^{*}(L)}{e}
\end{array},\right.
$$

where $i$ is the number of the virtual field used to built up the linear system and $\mathbf{X}$ is a vector containing the five unknowns.

Another solution is to consider more than one testing configuration. Some examples of these two approaches are presented and discussed below. Note finally that the shear stress-strain response only approximately fits a cubic response in practice. The actual response would be best 
fitted with a function including more monomials than the two ones which have been used in the present study (the linear and the cubic ones). These additional unknowns (the coefficients of the new monomials) could also be determined with additional equations in the linear system, but this problem is not examined here.

\section{Identification with five virtual fields and one testing configuration}

\subsection{Introduction}

The natural idea is first to consider again the four virtual fields in Table 1 that have been shown in Ref. [26] to be suitable for the determination of the parameters in the case of a linear shear response. If only one actual strain field is processed, the fifth field, which completes this set of four equations, must be independent of the others. It must be kinematically admissible and chosen in such a way that the actual stress field inside the grips does not virtually work. Hence, the virtual strain components inside the grips will be zero, and the corresponding virtual displacement must therefore be solidrigid like in this part of the specimen. This constraint must be taken into account for the construction of the virtual field in the active area of the specimen. Apart from these obvious constraints, no systematic rule is available to establish automatically this field from the four others and, at the present stage of the development of the method, the relevance of any new field can only be checked a posteriori.

\subsection{Finite element simulation of the identification method}

Since only the theoretical and numerical aspects of the approach are discussed in this work, the data to be processed are provided by a finite element programme. The CASTEM 2000 package has been used because it is well suited to nonlinear material behaviours. The nonlinear stress/strain constitutive Eq. (4) with the same stiffness values as in Ref. [26] has been programmed: $E_{x x}=25 \mathrm{GPa}, E_{z z}=$ $10 \mathrm{GPa}, \nu_{x z}=0.3, G_{x z}=4 \mathrm{GPa}$ with an additional term that governs the nonlinearity: $K=4420 \mathrm{GPa}$. Since the cubic law is not sufficient to describe the whole actual stress/strain curve (see Fig. 6): it is followed for these simulations by a straight line with a weak slope arbitrarily taken as $Q_{r r} / 7=Q_{x z} / 7$. The specimen in Fig. 1 is meshed with 23,520 triangular linear elements. It has been checked with a convergence study that the mesh density is sufficient. The boundary conditions are such that the contact between the grips and the specimen is unilateral. This is obtained after some iterative calculations, by relaxing at each step the nodes where the force between the grip and the specimen leads to a local traction of the specimen. For all the nodes in contact with the grip, that is to say those for which the force between the grip and the specimen leads to a local compression of the specimen, the imposed vertical displacement at the right-hand side (respectively, left-hand side) grip is constant and equal to $u_{z}=1.4 \mathrm{~mm}$ (respectively, $0 \mathrm{~mm}$ ). The strain components are collected at the gravity centre of the triangular elements located in the active area. These data are considered as input data for the identification procedure. It must be pointed out that some local stress concentrations occur near the edges of the grips. Some shear strain components are therefore greater than $\epsilon_{r_{\max }}$ in Fig. 6 and cannot be considered as proper input data for determining the parameters of the cubic law. Hence the elements where the shear strain is greater than $\epsilon_{r_{\max }}$ are removed from the collected data. For practical reasons, not only the areas near the edge of the grips

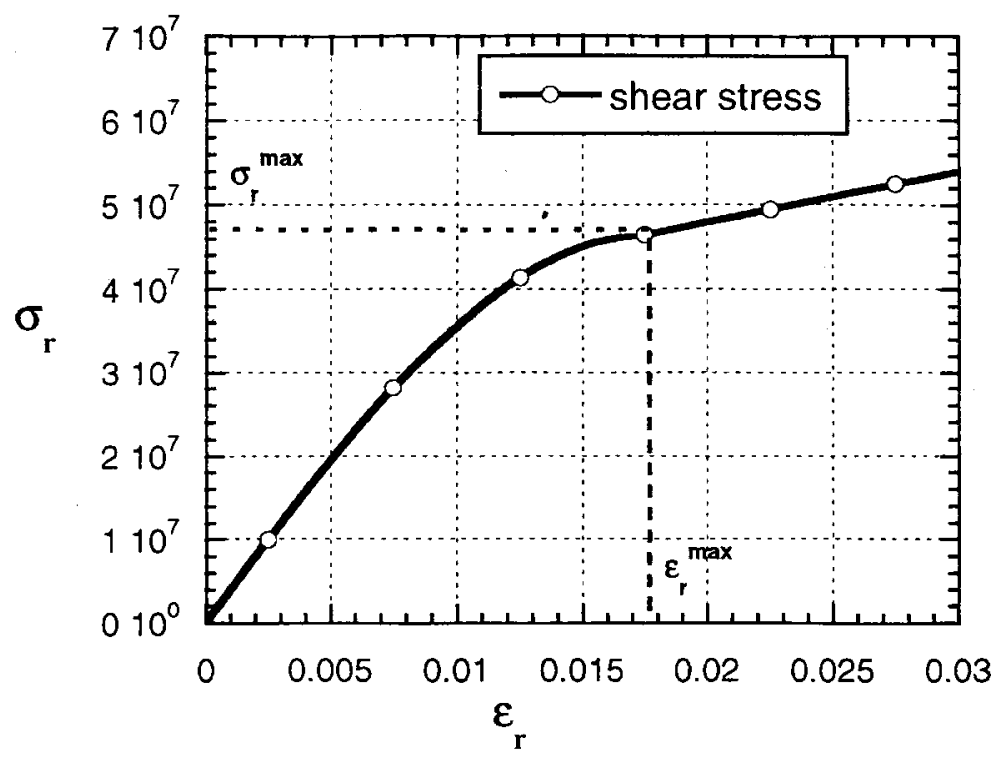

Fig. 6. Nonlinear shear response of the constitutive material. 


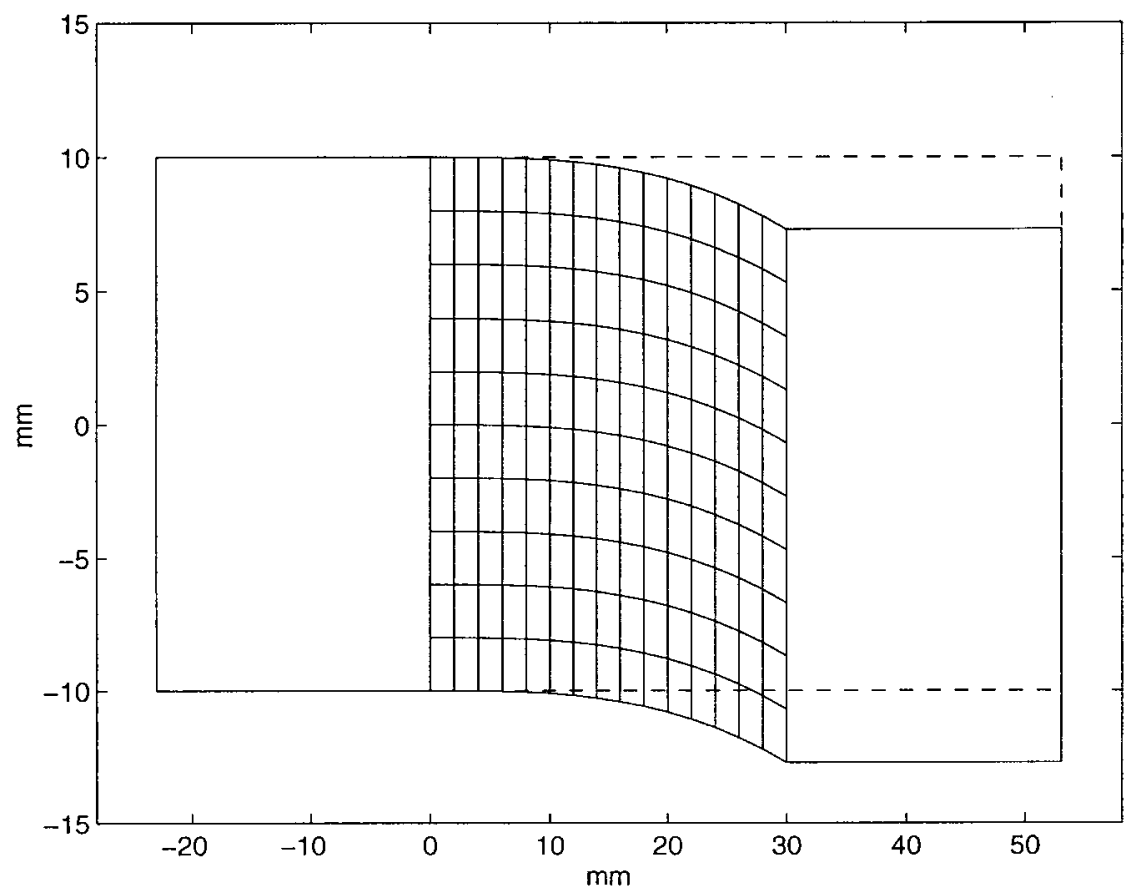

Fig. 7. Virtual field 5: virtual cubic vertical displacement.

but also the whole columns of elements containing these areas were removed. The area where the actual strain field is processed remains therefore rectangular, but it is about $80 \%$ of the initial active area.

As explained above, for this simulation, the imposed displacement is $0 \mathrm{~mm}$ at the left-hand grip and $u_{z}=$ $1.4 \mathrm{~mm}$ at the right-hand grip. Those values are adjusted in such a way that the maximum shear strain at the centre of the active area is about $0.95 \times \epsilon_{r_{\max }}$. It can be checked that $L$ has been adjusted in such a way that the three strain components $\sigma_{x}, \sigma_{z}$ and $\sigma_{r}$ are roughly of equal magnitude in the active area away from the local stress concentrations, leading to a possible identification of the material parameters. The vertical forces at the nodes where the displacement $u_{z}$ is imposed are collected and added together to provide the global resultant vertical force $F$ that would be measured in practice by the load cell of the testing machine. The right-hand side in Eq. (5) can therefore be easily calculated. It must be pointed out that the nodes where the displacements are imposed are chosen in such a way that the contact between grip and specimen remains locally unidirectional.

Only the resultant of the external load on the right-hand grip can be measured in practice whereas the loading distribution on the boundary of the grip cannot be assessed. Consequently it is proposed to construct the fifth virtual field in such a way that the right-hand side grip virtually moves like a rigid solid in the vertical direction, without any virtual rotation. The trial-and-error approach performed to construct the fifth virtual field is illustrated by the two examples below.

\subsection{Virtual cubic vertical displacement}

\subsubsection{Construction and results}

As a first attempt, a polynomial field such that $u_{x}^{*}=$ 0 and $u_{z}^{*}=x^{n}$ is considered. This type of field is an extension of the first field in Table 1 where $n=1$. The results below are given in the case $n=3$. Other powers $n$ were tested but they led to similar results: the corresponding results are therefore not reported here. This virtual field is plotted in Fig. 7. The parameters identified in this case are reported in Table 2. It is worth noting that the identified values are very close to the actual ones, apart from $Q_{x z}$ which is not identified correctly, contrary to the results in Ref. [26], where this term is only rather sensitive to noise in the data. A possible reason is the fact that the active area has been reduced to eliminate the columns of elements where the shear strain is greater than $\epsilon_{r_{\max }}$. As a general remark, note also that $Q_{x z}$ often only slightly influences the actual strain/stress fields. This leads in practice to some inaccurate results concerning this coefficient or to an important sensitivity to noisy data, as reported in Refs. $[6,14,15]$ for instance, where other numerical strategies than the present

Table 2

Virtual cubic vertical displacement. Reference and identified values for the material parameters

\begin{tabular}{lccccc}
\hline & $Q_{x x}$ & \multicolumn{1}{c}{$Q_{z z}$} & \multicolumn{1}{c}{$Q_{x z}$} & $Q_{r r}$ & $K$ \\
\hline Reference (GPa) & 25.94 & 10.37 & 3.11 & 4.00 & 4420 \\
Identified (GPa) & 25.49 & 10.00 & 0.77 & 4.00 & 4279 \\
Relative difference (\%) & -1.7 & -3.6 & -75 & 0.0 & -3.2
\end{tabular}


Table 3

Virtual cubic vertical displacement. Identified stiffness distribution from errors simulations on the strains

\begin{tabular}{|c|c|c|c|c|c|}
\hline & $Q_{x x}$ & $Q_{z z}$ & $Q_{x z}$ & $Q_{r r}$ & $K$ \\
\hline Reference (GPa) & 25.94 & 10.37 & 3.11 & 4.00 & 4420 \\
\hline \multicolumn{6}{|l|}{$5 \%$} \\
\hline Identified (GPa) & 25.52 & 9.91 & 0.78 & 4.00 & 4281 \\
\hline Relative difference $(\%)$ & -1.6 & -4.5 & -74.8 & $0.0\}$ & -3.1 \\
\hline Coefficient of variation $(\%)$ & 0.2 & 2.4 & 8.5 & 0.3 & 1.4 \\
\hline \multicolumn{6}{|l|}{$10 \%$} \\
\hline Identified $(\mathrm{GPa})$ & 25.55 & 9.83 & 0.8 & 4.00 & 4292 \\
\hline Relative difference $(\%)$ & -1.5 & -5.2 & -74.3 & 0 & -2.8 \\
\hline Coefficient of variation $(\%)$ & 0.2 & 4.7 & 16.6 & 0.5 & 2.8 \\
\hline
\end{tabular}

one were used. This drawback could certainly be reduced by choosing virtual fields that better highlight the influence of this coefficient (even if it is small in the actual field), but this difficult question is not examined here.

Since the present approach is designed to be used with experimental data, it is of prime importance to examine the stability of the four remaining parameters with respect to noise in the data. Two cases are considered. First, a random noise is added to each of the strain components of the field. Since the displacement/strain field will be captured by a CCD camera in practice, the effect of a global shift of the camera is examined in a second simulation.

\subsubsection{Random noise}

In the first simulation, a random noise denoted $\delta$ is added to each of the three simulated strain components provided by the FE programme

$\left\{\begin{array}{l}\epsilon_{x}^{\prime}=\epsilon_{x}+\delta \\ \epsilon_{z}^{\prime}=\epsilon_{z}+\delta \\ \epsilon_{r}^{\prime}=\epsilon_{r}+\delta\end{array}\right.$

The average of the noise $\delta$ is zero. Its distribution is uniform between two bounds which are successively \pm 5 and $\pm 10 \%$ of the maximum of each strain component in the active area. The distribution of the noise is uniform between the two bounds. Repeating the identification procedure 30 times leads to a distribution of the disturbed identified stiffnesses. The average and coefficients of variation are reported in Table 3. The coefficient of variation is computed in each case as an indicator of the scatter, even though the distribution is linear and not normal. It can be seen that the shear stiffness is the most stable. On the other hand, $Q_{x z}$ is the less stable. The sensitivity of the other terms is low. These results are in agreement with the results found above. This relative global stability is due to the average effect caused by the integrals in Eq. (5), which tends to average out local discrepancies between exact and disturbed strain components. This feature is one of the main advantages of the present method.

\subsubsection{Global shift of the $x$ - and z-coordinates}

A global shift is now considered. A constant value is added to each coordinate $x$ and $z$ of the simulated captured data points in order to simulate an imperfect centring of the camera on the specimen. Let us assume that the size of the CCD grid of the camera is $512 \times 512$, the step between two pixels of the grid is equal to $30 \mathrm{~mm} / 512$. Hence, if the shift is equal to $m$ pixels, a constant value equal to $m \times 30 / 512 \mathrm{~mm}$ is added to each coordinate. Results obtained in two cases: $m=6$ and $m=12$ are reported in Table 4. It clearly appears that the procedure is unstable in the present case: a slight variation of some of the coefficients in matrix A leads to a very important variation of the identified values. The reason is that the five equations are almost dependent.

\subsubsection{Analysing the dependence of the linear equations}

Before analysing the dependence of the linear equations, a 'scaling' of the matrix A has been carried out. Since the magnitude of the components in matrix $\mathbf{A}$ are not of the same order, each row is divided by its highest value. This leads in practice to a multiplication of matrix $\mathbf{A}$ by a matrix denoted $\mathbf{Q}_{1}$. In the diagonal of $\mathbf{Q}_{1}$ are reported the inverse of the maxima of each row in matrix A. Furthermore, since the magnitude of the five unknowns is very different because of $K$, each column of the product $\mathbf{Q}_{1} \mathbf{A}$ is divided by its maximum. This leads in practice to a multiplication of $\mathbf{Q}_{1} \mathbf{A}$ by a matrix $\mathbf{Q}_{2}$ which diagonal is made of the inverse of the maxima of each column. Finally, the initial matrix $\mathbf{A}$ is replaced by $\mathbf{A}^{\prime}=\mathbf{Q}_{1} \mathbf{A} \mathbf{Q}_{2}$ and the solution is found by computing $\mathbf{X}=\mathbf{Q}_{2}\left(\mathbf{A}^{\prime-1} \mathbf{Q}_{1}\right) \mathbf{B}$. The main advantage is the

Table 4

Virtual cubic vertical displacement. Influence of a shift

\begin{tabular}{|c|c|c|c|c|c|}
\hline & $Q_{x x}$ & $Q_{z z}$ & $Q_{x z}$ & $Q_{r r}$ & $K$ \\
\hline Reference (GPa) & 25.94 & 10.37 & 3.11 & 4.00 & 4420 \\
\hline Identified (GPa) & 25.49 & 10.00 & 0.77 & 4.00 & 4279 \\
\hline Relative difference $(\%)$ & -1.7 & -3.6 & -75 & 0.0 & -3.2 \\
\hline Shift, $m=6(\mathrm{GPa})$ & 25.79 & 49 & 2.2 & -7.3 & $-61,376$ \\
\hline Relative difference $(\%)$ & -0.5 & $\geq 100$ & -28.7 & $\leq-100$ & $\leq-100$ \\
\hline Shift, $m=12(\mathrm{GPa})$ & 26.29 & 90.4 & 4.61 & -18.8 & $-128,458$ \\
\hline Relative difference $(\%)$ & 1.3 & $\geq 100$ & 48.3 & $\leq-100$ & $\leq-100$ \\
\hline
\end{tabular}


Table 5

Angles $\theta_{i}$ between vectors $\mathbf{V}_{i}$ characterizing the equations of the linear system

\begin{tabular}{lllll}
\hline Fifth field & $\theta_{1}\left({ }^{\circ}\right)$ & $\theta_{2}\left({ }^{\circ}\right)$ & $\theta_{3}\left({ }^{\circ}\right)$ & $\theta_{4}\left({ }^{\circ}\right)$ \\
\hline Field 5 & 90 & 38.7 & 36.4 & 0.3 \\
Field 6 & 90 & 38.7 & 36.4 & 2.9 \\
\hline
\end{tabular}

fact that the components in matrix $\mathbf{A}^{\prime}$ are of comparable magnitude.

The degree of independence of each equation in the linear system is now assessed as follows. The $i$ th equation in the new linear system can be characterized by a vector denoted $\mathbf{V}_{i}$ which components are

$\mathbf{V}_{i}:\left(\begin{array}{c}\mathbf{A}_{i 1}^{\prime} \\ \mathbf{A}_{i 2}^{\prime} \\ \cdots \\ \mathbf{A}_{i 5}^{\prime}\end{array}\right)$

This vector is perpendicular to the hyperplane which equation is the $i$ th equation in the system. The idea is to compute the angle $\theta_{i}$ between each vector $\mathbf{V}_{i}+1$ and the space built up with the vectors $\mathbf{V}_{k}, k=1 \ldots i-1$. Obtaining a set of angles all equal to $90^{\circ}$ means that the equations are completely independent (all hyperplanes are perpendicular in this case). If one of the angles is equal to zero, the equations are dependent. Between these two cases, a small angle means that the equations are 'almost' dependent. This set of angle is a practical indicator to compare different linear systems provided by different sets of virtual fields and to assess the quality of the linear systems to be inverted. The angles found in the present case are reported in the first row in Table 5. It clearly appears that the fourth angle is very close to zero, showing that the fifth field does not lead to an equation 'sufficiently' independent of the four others.

The reason for that comes from the actual stress field itself and can be explained as follows. Let us consider a virtual displacement field such that $u_{x}^{*}$ is zero and $u_{z}^{*}$ only depends on the $x$-coordinate, like the above fifth virtual field. Hence $u_{z}^{*}=u_{z}^{*}(x)$ and consequently, $\epsilon_{r}^{*}=$ $\epsilon_{r}^{*}(x)$. Let us now assume that the actual shear stress field only depends on the $z$-coordinate. Hence, $\sigma_{r}=$ $\sigma_{r}(z)$ and $\epsilon_{r}=\epsilon_{r}(z), \epsilon_{x}=\epsilon_{z}=0$. We have approximately such an actual shear stress field in our case, as could be checked in the shear stress distribution. In this case, the equilibrium Eq. (5) reduces to

$$
\begin{aligned}
& Q_{r r} \int_{-h / 2}^{h / 2} \int_{0}^{L} \epsilon_{r}(z) \epsilon_{r}^{*}(x) \mathrm{d} x \mathrm{~d} z-K \int_{-h / 2}^{h / 2} \int_{0}^{L} \epsilon_{r}^{3}(z) \epsilon_{r}^{*}(x) \mathrm{d} x \mathrm{~d} z \\
& \quad=-\frac{P u_{z}^{*}(L)}{e}
\end{aligned}
$$

$$
\begin{aligned}
& \epsilon_{r}^{*}(x)=u_{z, x}^{*}(x) \text { in our case, thus } \\
& Q_{r r} \int_{-h / 2}^{h / 2} \int_{0}^{L} \epsilon_{r}(z) \mathrm{d} z \int_{0}^{L} u_{z, x}^{*}(x) \mathrm{d} x \\
& \quad-K \int_{-h / 2}^{h / 2} \epsilon_{r}^{3}(z) \mathrm{d} z \int_{0}^{L} u_{z, x}^{*}(x) \mathrm{d} x \\
& \quad=-\frac{P u_{z}^{*}(L)}{e}
\end{aligned}
$$

and finally, after integration in the $x$-direction

$$
u_{z}^{*}(L)\left(Q_{r r} \int_{-h / 2}^{h / 2} \epsilon_{r}(z) \mathrm{d} z-K \int_{-h / 2}^{h / 2} \epsilon_{r}^{3}(z) \mathrm{d} z\right)=-\frac{P u_{z}^{*}(L)}{e} .
$$

As can be seen, feeding in the above equation any virtual field $u_{z}^{*}$ verifying the above requirements and such that $u_{z}^{*}(L) \neq 0$ leads in any case to the same equation since $u_{z}^{*}(L)$ appears in both sides in the above equation. Since we used $u_{z}^{*}=x$ and $u_{z}^{*}=x^{3}$ as, respectively, first and fifth equations to built up the linear system, we have finally obtained two equations that are almost dependent since the actual shear stress field almost only depends on the $z$-coordinate. The solution obtained is therefore unstable. This has been clearly observed above when the components of the matrix are slightly modified. Let us now built up another fifth field expected to lead to a better independence of the equations.

\subsection{Virtual field with a parabolic virtual shear strain distribution through the thickness}

\subsubsection{Construction of the field}

The objective is here to propose an alternative virtual field that will lead to a fifth independent equation. Since the first field led to a constant virtual shear strain in the active part, the idea is to construct a virtual field that depends on $z$. A linear virtual shear strain would not be relevant. This is due to the fact that the integral that provides the coefficient of $Q_{r r}$ and $K$ in Eq. (5) would be zero because of the evenness of the integrand. On the other hand, a quadratic virtual shear strain would not lead to zero coefficient. One could therefore suggest the following virtual shear strain

$\epsilon_{r}^{*}=z^{2}-\frac{h^{2}}{4}$,

where $h$ is the height of the specimen. Such a shear strain leads to a maximum value at the centre of the specimen and is like the shear strain field distribution in higher-order theories for thick structures. The contribution of the actual shear stress at the centre of the specimen is expected to be maximum. The feature is important since the actual shear stress is also maximum at the centre of the specimen. It is therefore expected to magnify the influence of the nonlinearity 


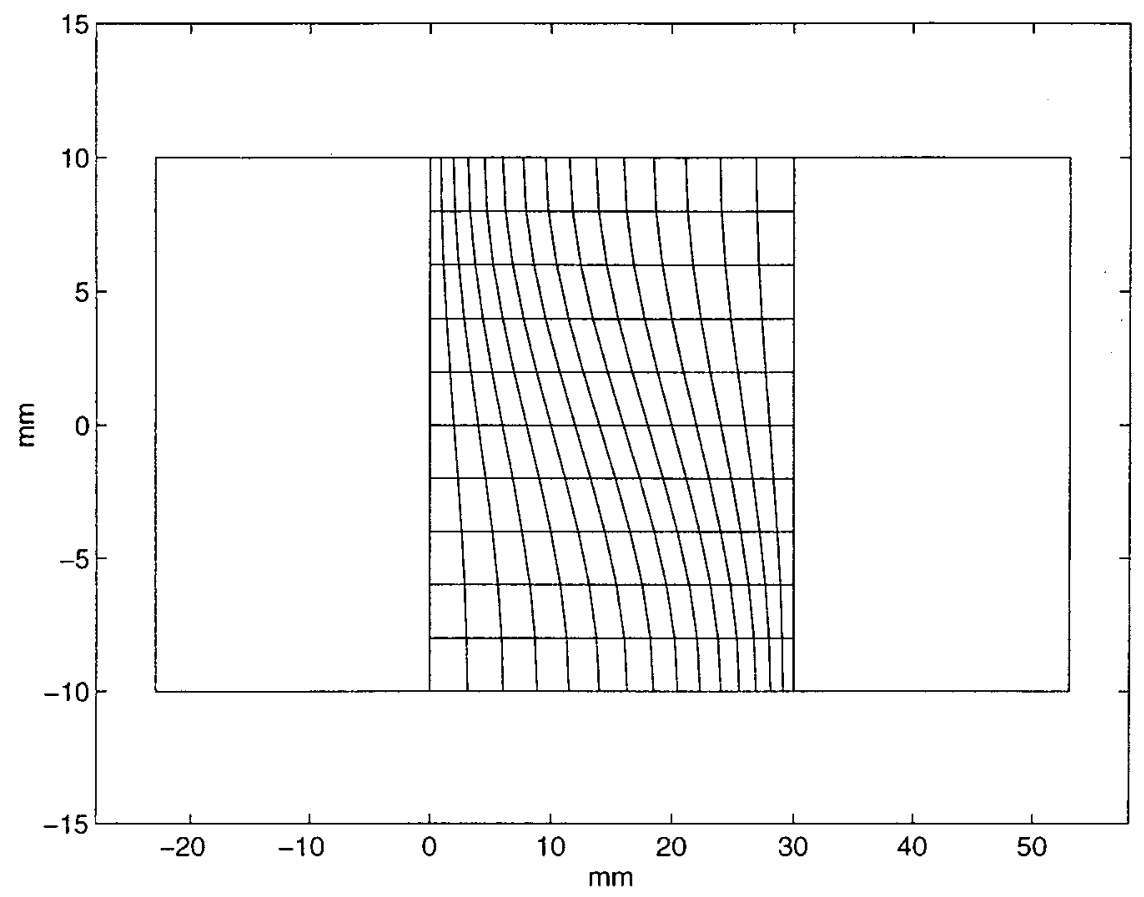

Fig. 8. Virtual field 6: virtual parabolic through-thickness shear strain.

compared to the first field, for which the virtual shear strain is constant. Considering a quadratic shear strain leads however to a warping of the sections, but this warping must decrease and reach zero near the grips, at the boundary of the active part of the specimen, since the virtual displacement field within the grips must be solid-rigid-like. It is therefore suggested to weight the quadratic virtual shear strain as follows

$\epsilon_{r}^{*}=x(x-L)\left(z^{2}-\frac{h^{2}}{4}\right)$.

The virtual field is easily obtained by integration

$$
\left\{\begin{array}{l}
u_{x}^{*}=x(x-L) z\left(\frac{z^{2}}{3}-\frac{h^{2}}{4}\right) . \\
u_{z}^{*}=0
\end{array}\right.
$$

This virtual field is plotted in Fig. 8. It can be checked that the corresponding virtual vertical displacement of the right- hand side grip is zero, leading to a zero virtual work of the external loading. A non zero virtual strain $\epsilon_{x}^{*}$ appears by differentiation of the virtual displacement and the final virtual strain field is

$$
\left\{\begin{array}{l}
\epsilon_{x}^{*}=(2 x-L) z\left(\frac{z^{2}}{3}-\frac{h^{2}}{4}\right) \\
\epsilon_{z}^{*}=0 \\
\epsilon_{r}^{*}=x(x-L)\left(x^{2}-\frac{h^{2}}{4}\right)
\end{array} .\right.
$$

\subsubsection{Results and discussion}

The above virtual field has been used in the linear system and the identification procedure has been applied with the simulated data provided by the FE computations. Results are reported in Table 6 . When no error is considered, the accuracy is about the same as in the preceding case. The sensitivity to a global shift is however much lower. This

Table 6

\begin{tabular}{|c|c|c|c|c|c|}
\hline & $Q_{x x}$ & $Q_{z z}$ & $Q_{x z}$ & $Q_{r r}$ & $K$ \\
\hline Reference (GPa) & 25.94 & 10.37 & 3.110 & 4.000 & 4420 \\
\hline Identified (GPa) & 25.49 & 10.00 & 0.8 & 4.00 & 4270 \\
\hline Relative difference $(\%)$ & -1.7 & -3.6 & -75.4 & 0.0 & -3.4 \\
\hline Shift, $m=6(\mathrm{GPa})$ & 25.75 & 10.74 & 2.02 & 3.96 & 4060 \\
\hline Relative difference $(\%)$ & -0.7 & 3.6 & -35.1 & -0.9 & -8.1 \\
\hline Shift, $m=12(\mathrm{GPa})$ & 25.60 & 12.29 & 1.3 & 3.89 & 3665 \\
\hline Relative difference $(\%)$ & -1.3 & 18.5 & -59.5 & -2.6 & -17.0 \\
\hline
\end{tabular}

Virtual parabolic through-thickness shear strain. Reference and identified values for the material parameters in different cases 
feature is directly related to the angle $\theta_{4}$ introduced above. This angle is reported in Table 5, row 2. As can be seen, the value of this angle is higher than in the preceding case, but it is clearly the lowest of the four angles. The consequence appears in Table 6, where one can observe that the sensitivity of $Q_{r r}, Q_{x z}$ and $K$ to noise in the data has been improved since it is lower than in the previous case. It is however greater than that obtained when the elastic parameters only are identified [26].

\subsection{Conclusion}

Several tracks can be followed to improve the above results:

- searching other more relevant virtual fields but this critical point is not considered here;

- the removal of elements that reduces the processed area has probably an harmful effect for the evaluating the correct value of $Q_{x z}$. Indeed, in Ref. [26], the correct value of $Q_{x z}$ is obtained by using the same method with the following conditions: the material is linear elastic and the area $S_{2}$ is the whole processed area. In practice, one could therefore estimate properly $Q_{x z}$ by using the approach developed in Ref. [26] at the beginning of the loading and the present one when the nonlinearity occurs, that is when the actual shear strain becomes close to $\epsilon_{r_{\max }}$ near the grips. As a result, the five parameters would be accurately identified;

- another way to feed the linear system with some new equations is to consider other actual stress fields. If the constitutive material is linear elastic, stress fields provided by other testing configurations should also be processed. In the present case of a non-linear shear response, one can consider the same specimen under increasing load levels. The strain/stress fields are not proportional from one level to another and one can expect to obtain some new independent equations. This point is examined in the next section.

\section{Identification with four virtual fields and several testing configurations}

\subsection{Introduction}

Since the first four virtual fields have been shown to be satisfactory to identify the elastic constants, they are kept to build the first four equations of the linear system with the actual stress/strain field provided by the model with an imposed displacement of the right-hand grip equal to $u_{z}=$ $1.4 \mathrm{~mm}$. The strain components at the nodes are also collected at intermediate values of the displacement of the grip. These values are processed with the simplest virtual field, that is the first in Table 1. A new equation is obtained at each load level. Two cases are presented below. In the
Table 7

Values obtained after processing the actual fields obtained at two loading levels

\begin{tabular}{lccccc}
\hline & $Q_{x x}$ & $Q_{z z}$ & $Q_{x z}$ & $Q_{r r}$ & $K$ \\
\hline Reference (GPa) & 25.94 & 10.37 & 3.11 & 4.00 & 4420 \\
Identified (GPa) & 25.49 & 10.15 & 0.8 & 3.96 & 4016 \\
Relative difference $(\%)$ & -1.7 & -2.1 & -75 & -1.1 & -9.1 \\
Shift, $m=6(\mathrm{GPa})$ & 25.76 & 10.77 & 2.02 & 3.96 & 4016 \\
Relative difference $(\%)$ & 0.7 & 3.8 & -35 & -1.1 & -9.1 \\
Shift, $m=12(\mathrm{GPa})$ & 25.59 & 12.08 & 1.25 & 3.96 & 4016 \\
Relative difference $(\%)$ & 1.3 & 16.5 & -59.8 & -1.1 & -9.1 \\
\hline
\end{tabular}

first case, the fifth equation is obtained with the strain field at $u_{z} / 2=0.7 \mathrm{~mm}$. In the second case, a set of actual fields obtained at different imposed displacements is processed with the first virtual field to obtain a redundant linear system.

\subsection{Two load levels}

As explained above, the actual shear strain field in the active area is collected at $u_{z} / 2$ and processed with the first virtual field to obtain the fifth linear equation. The values obtained after inversion of the system are reported in Table 7. The global shift with $m=6$ and $m=12$ is also performed. The results are slightly better than in the preceding case. The two shear parameters $Q_{r r}$ and $K$ are stable with respect to the shift. This is certainly due to the fact that the first virtual field is linear. Then, the corresponding strain components are constant and therefore not sensitive to any additional shift in the coordinate of the point.

\subsection{Six loading levels}

As a final example, the actual shear strain field in the active area is collected at $u_{z} / 4, u_{z} / 3, u_{z} / 2,2 u_{z} / 3$ and $3 u_{z} / 4$ and processed with the first virtual field to obtain five linear equations that complete the four first ones obtained with the actual strain fields at $u_{z}$. A linear system of nine equations with five unknowns is finally obtained. It is solved with the least square method: the initial system $\mathbf{A X}=\mathbf{B}$ is multiplied by the transposed matrix of $\mathbf{A}$ denoted $\mathbf{A}^{\prime}$ to obtained a linear system of five equations with five unknowns $\mathbf{A}^{\prime} \mathbf{A} \mathbf{X}=$ $\mathbf{A}^{\prime} \mathbf{B}$. This linear system is easily inverted: $\mathbf{X}=\left(\mathbf{A}^{\prime} \mathbf{A}\right)^{-1} \times$ $\left(\mathbf{A}^{\prime} \mathbf{B}\right)$. The results are reported in Table 8. Except again for

Table 8

Values obtained after processing the actual fields obtained at six loading levels

\begin{tabular}{lccccc}
\hline & $Q_{x x}$ & $Q_{z z}$ & \multicolumn{1}{c}{$Q_{x z}$} & \multicolumn{1}{c}{$Q_{r r}$} & $K$ \\
\hline Reference $(\mathrm{GPa})$ & 25.94 & 10.37 & 3.11 & 4.000 & 4420 \\
Identified $(\mathrm{GPa})$ & 25.53 & 10.09 & 0.93 & 3.98 & 4162 \\
Relative difference $(\%)$ & -1.6 & -2.7 & -70 & -0.4 & -5.8 \\
Shift, $m=6(\mathrm{GPa})$ & 25.79 & 10.71 & 2.19 & 3.98 & 4162 \\
Relative difference $(\%)$ & 0.1 & 3.3 & -29.7 & -0.4 & -5.8 \\
Shift, $m=12(\mathrm{GPa})$ & 26.63 & 12.03 & 1.42 & 3.98 & 4162 \\
Relative difference $(\%)$ & -1.1 & 16.0 & -54.2 & -0.4 & -5.8
\end{tabular}


the $Q_{x z}$ term which is completely wrong, the parameters are close to the reference values. Improving the number of actual fields to be processed has also improved the accuracy on the nonlinear term $K$. The stability of this term also clearly appears. In conclusion of the above results, it seems that processing more than one mechanical configuration leads to better results than processing only one mechanical configuration with the fifth virtual fields presented in Section 3.4.

\section{Conclusion}

The method described here allows the determination of the through-thickness properties of a thick composite including a nonlinear shear response. The method is based on the use of the principle of virtual work with particular virtual fields. Actual strain fields onto the central part of the specimen are processed. Such fields should be obtained in practice with suitable optical techniques.

Two strategies have been presently tested. First, only one mechanical configuration has been considered. The main problem that occurs is to build an independent virtual field, which completes the four fields suitable for determining elastic parameters. Another solution is to process strain fields obtained at different loading levels. This latter procedure has been shown to be more accurate and stable than the previous one.

In both approaches, the shear properties are identified with the highest accuracy and stability. The probable reason is the fact that the loading is obtained with a Iosipescu fixture initially developed for composite shear testing. On the other hand, the $Q_{x z}$ stiffness could not be identified. Previous work carried out within the framework of linear elasticity does not confirm this results [26]. This is probably due to the fact that a part of the active area of the specimen near the grips has been presently removed for the processing because the shear strain at some points is not modelled by the cubic law. One could avoid the present drawback by using in practice the approach developed in Ref. [26] at the beginning of the loading to obtain precisely $Q_{x z}$ and the present one when the nonlinearity occurs, that is when the actual shear strain becomes close to $\epsilon_{r_{\max }}$ near the grips to obtain precisely $K$. As a result, the five parameters would be accurately identified.

Two main issues remain to be addressed in the near future. First, the experimental implementation of the approach has to be carried out. Second, it should be relevant to find a systematic method to build up virtual fields leading to independent equations. Indeed, it is clear that any significant improvement of the virtual fields method is now related to the determination of independent and optimal virtual fields.

\section{References}

[1] Gipple KL, Hoyns D. Measurement of the out-of-plane shear response of thick section composite materials using the V-notched beam specimen. J Compos Mater 1994;28(6):543-72.

[2] Mespoulet S. Through-thickness test methods for laminated composite materials. PhD thesis, Imperial College of Science and Medicine, London, UK, 1998.

[3] Broughton W, Lodeiro MJ, Sims GD. Understanding the limitations of through-thickness test methods. In: Proceedings of the Fourth European Conference on Composites: Testing and Standardisation, 31 August-2nd September in Lisbon, Portugal, 1998;80-90.

[4] Broughton W. An overview of through-thickness test methods for polymer matrix composites. Technical report, National Physical Laboratory DMM(A) 148, 1994.

[5] Report from exploratory phase and full network proposal thematic network. Standard Measurements and Testing, 4th framework programme, 1997.

[6] Sol H. Identification of anisotropic plate rigidities using free vibration data. Doctoral dissertation, University of Brussels, 1986.

[7] De Wilde WP. Identification of the rigidities of composite systems by mixed numerical/experimental techniques. Mechanical identification of composites, Amsterdam: Elsevier, 1990. p. 1-15.

[8] Hendricks MAN. Identification of the mechanical properties of solid materials. Doctoral dissertation, Eindhoven University of Technology, 1991.

[9] Pedersen P, Frederiksen PS. Identification of orthotropic materials moduli by combinated experimental numerical approach. Measurements 1992;10:113-8.

[10] Ayorinde EO, Gibson RF. Elastic constants of orthotropic composite materials using plate resonance frequencies, classical lamination theory and an optimized three-mode Rayleigh formulation. Compos Engng 1993;3(5):395-407.

[11] Deobald LR, Gibson RF. Determination of elastic constants of orthotropic plates by a modal analysis/Rayleigh-Ritz technique. J Sound Vibr 1988;124(2):269-83.

[12] Mota Soares CM, Moreira de Freitas M, Araújo AL, Pedersen P. Identification of material properties of composite plate specimens. Compos Struct 1993;25:277-85.

[13] Araujo AL, Mota Soares CM, Freitas MJM. Characterization of material parameters of composite specimens using optimization and experimental data. Compos Part B 1996;27(2):185-91.

[14] Frederiksen PS. Numerical studies for identification of orthotropic elastic constants of thick plates. Eur J Mech A/Solids 1997;16:11740.

[15] Frederiksen PS. Parameter uncertainty and design of optimal experiments for the estimation of elastic constants. Int J Solids Struct 1998; 12:1241-60.

[16] Cunha J, Piranda J. Application of model updating techniques in dynamics for the identification of elastic constants of composite materials. Compos Part B 1999;30:79-85.

[17] Rastogi PK. Photomechanics. Berlin: Springer, 1999.

[18] Grédiac M. Principe des travaux virtuels et identification/principle of virtual work and identification. Comptes Rendus de l'Académie des Sciences 1989;II(309):1-5 (Gauthier-Villars, in French with Abridged English Version).

[19] Grédiac M, Vautrin A. A new method of determination of bending rigidities of thin anisotropic plates. J Appl Mech 1990;57(December):964-8.

[20] Grédiac M. On the direct determination of invariant parameters governing the bending of anisotropic plates. Int J Solids Struct 1996;33(27):3969-82.

[21] Grédiac M, Paris P-A. Direct identification of elastic constants of anisotropic plates by modal analysis: theoretical and numerical aspects. J Sound Vibr 1996;195(3):401-15.

[22] Grédiac M, Fournier N, Paris P-A, Surrel Y. Direct identification of elastic constants of anisotropic plates by modal analysis: experiments and results. J Sound Vibr 1998;210(5):645-59.

[23] Grédiac M, Pierron F. A T-shaped specimen for the direct characterization of orthotropic materials. Int J Numer Methods Engng 1998;41:293-309. 
[24] Grédiac M, Pierron F, Surrel Y. Novel procedure for complete inplane composite characterization using a T-shaped specimen. Exp Mech 1999;39(2):142-9.

[25] Pierron F, Zhavaronok S, Grédiac M. Identification of the throughthickness properties of thick laminates using the virtual fields method Int J Solids Struct 2000;37(32):4437-53.

[26] Pierron F, Grédiac M. Identification of the through-thickness moduli of thick composites from whole-field measurements using the Iosipescu fixture: theory and simulations. Compos: Part A 2000;31(4):309-18.

[27] Okada H, Fukui Y, Kumazawa N. An inverse analysis determining the elastic-plastic stress-strain relationship using nonlinear sensitivities. Comput Model Simul Engng 1999;4(3):176-85.

[28] Bulhak J, Surrel Y. Grating shearography. In: Interferometry '99:
Techniques and Technologies, 20-23 September 1999, Pułtusk, Poland, vol. SPIE 3744, 1999;506-15.

[29] Surrel Y. Moiré and grid methods: a signal-processing approach. Interferometry '94: photomechanics. Pryputniewicz RJ, Stupnicki J, editors. Proc Soc Photo-Opt Instrum Engng 1994;2432:213-20.

[30] Swasson SR, Messick M, Toombes GR. Comparison of torsion tube and Iosipescu in-plane shear test results for a carbon epoxy composite. Composites 1985;16(3):220-4.

[31] Ho H, Morton J, Farley GL. Nonlinear numerical analysis of the Iosipescu specimen for composite materials. Compos Sci Technol 1985;50(3):355-65.

[32] Cerisier F. Etude d'une transmission composite et de ses liaisons. Doctoral dissertation, University of Lyon, in French with English abstract, 1997. 